(RESEARCH ARTICLE)

\title{
Fucoidan: A nutraceutical for metabolic and regulatory systems homeostasis maintenance
}

\author{
Emil Mukhamejanov * and Victor Kurilenko
}

Fucoidan World, Almaty, Kazakstan.

Publication history: Received on 18 April 2020; accepted on 28 April 2020

Article DOI: https://doi.org/10.30574/wjarr.2020.6.1.0106

\begin{abstract}
Sustentation of homeostatic processes is the basic principle of vital activity of organism. Even small swings of concentration of some metabolites in the blood, especially glucose, lead to a violation of course of metabolic processes and pose a threat to vival activity of organism. Neuro-hormonal system, cytokine system and number of cell signaling systems participate in regulation of rate of metabolic processes. The influence of pharmaceutical preparations on these regulation systems can promote the development of serious complications, so there is an active search of natural food compounds with high bioactivity. The greatest interest in this situation is fucoidan, which is sulfated polysaccaride of brown seaweed. It is shown that it 1) supports the work of the central nervous system by moderately reducing the death of cholinergic neurons and maintaining the level of dopamine, 2) interferes with the development of hormonal imbalance, 3) promotes a decrease in the level of inflammatory cytokines in the blood and maintenance of the concentration of proinflammatory, 4) improves the functioning of the insulin cascade signaling systems at the mTOR kinase level by activating the translation process, 5) leads to a decrease in free radicals (antioxidant) and the restoration of their level (a prooxidant), 6) supports the homeostasis of the coagulation system due to anticoagulant and procoagulant properties. In this way, fucoidan should be attributed to nutraceuticals that promote health, improve quality and longevity of a person.
\end{abstract}

Keywords: Fucoidan; Nutraceutical; Gomeostasis; Regulation

\section{Introduction}

Medications have serious side effects, thereafter in recent years more and more attention has been paid to finding compounds related to food substances with high biological activity - nutriceuticals (derived from the words nutriology - nutrition and pharmaceutics). An ancient thinker, Hippocrates, wrote, "Our nutritional substances must be a healing agent (medication), and our medications must be nutritional substances.”

Life came out of the sea and all active components of the land flow into the seas back, therefore half of all bioactive compounds are present in the ecosystems of the seas. The sea algae, that contain such bioactive compounds as omega3, polyphenols, polysaccharides, fucosterol, and fucoxanthin, are of great interest in this regard. [1]. The diet of the inhabitants of Japan and South Korea in large quantities are present of dishes with brown algae, that is associated with their high life expectancy and low levels of cardiovascular disease and cancer. [2]. The main active component of the sea algae are polysaccharides, in particular, fucoidan, laminafaren, and alginate. In this review, we will focus on the fucoidan, sulfated polysaccharide that is also called fukan, fukosan or fukan sulfate. For the sea algae, fucoidan is a kind of "chain mail" for protection against biological factors (bacteria), mechanical damage (sand, waves), and physical effects (drying).

Fucoidan is synthesized in the cell wall of algae; therefore, to isolate the polysaccharide, fucoidan must be destroyed by hot water, organic solvents, or acids. Kylin used acetic acid for the extraction of fucoidan [3]. Later, he found that the

\footnotetext{
* Corresponding author: Emil Mukhamejanov
} 
polysaccharide fucoidan mainly contains L-fucose [4] that differs from other monosaccharides in its structure: it resembles galactose with no hydroxyl $(\mathrm{OH})$ group at 6 carbon atom and in L configuration (the remaining monosaccharides have the D configuration). Along with L-fucose, a large content of calcium and sulphate was also detected in the extract of fucoidan [5].

Structurally, fucoidans can vary significantly depending on the type of algae, the growing region, the harvest season and even the isolation methods. [6-7]. In this regard, fucoidan can not be called a medicine (those one have a strict chemical structure), and it is necessary to be referred as food compounds of plant origin.

The bioactivity of fucoidan largely depends on the content of L-fucose and sulphate groups [8]. The content of fucose can vary from 19 to $51 \%$, and sulfates from 19 to $43 \%$, depending on the type of algae and methods for isolating the polysaccharide. [9].

Separation of fucoidan (usually all fucoidan preparations consist of a mixture of polysaccharides of different size and charge) using ion-exchange chromatography into three fractions (F1, F2 and F3) showed differences in their antioxidant activity [10], which were $83.7,81.7$ and $41.6 \mathrm{mg} / \mathrm{g}$ for these fractions, respectively.

The presence of acyl groups has an important effect on the bioactivity of fucoidan [11], they are responsible for the hydrophilic properties of fucoidan (ability to swell or bind water).

The size of the fucoidan molecule affects the site of action of the polysaccharide and its bioactivity. [12]. The smaller the molecular size of fucoidan, the greater its amount can flow from the gastrointestinal tract into the bloodstream. Since humans do not have the enzymes to hydrolyze fucoidan (fucosidases), they can only enter the bloodstream through pinocytosis, and in this way smaller molecules or lower molecular weight (LM) fractions of fucoidan are better penetrated.

The structure of fucoidan has a key effect on the activity of the polysaccharide [13]. Fucoidan isolated from Fucus vesiculosus is a commercial medicine (Biotech company). It contains $44.1 \%$ fucose, $26.3 \%$ sulphate and $31.1 \%$ ash. It contains $44.1 \%$ fucose, $26.3 \%$ sulphate and $31.1 \%$ ash. [14]. Based on infrared data, it is shown that at the C-4 position, fucoidan is L-fucopyranose [15]. In 1993, Pankter et al. presented a structural model of fucoidan (Fig. 1), which is a polymer of $\alpha-(1 \rightarrow 3)$ bonds of fucose residues with branched chains with 2-3 fucose residues [16].

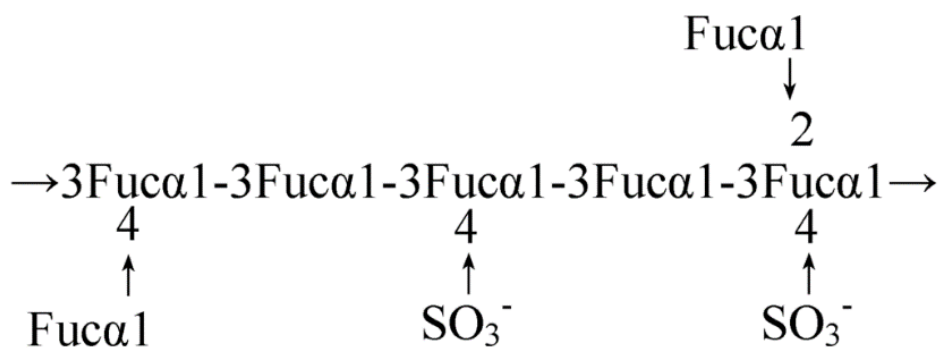

Figure 1 Model of the structure of fucoidan

The compounds absorbed from the intestines enter the liver with blood, and only then can they enter the general circulation, therefore, the assessment of fucoidan content in the liver is a marker of the flow of polysaccharide into the bloodstream. The liver is a "fasting block" to control compounds from the intestine. It was shown by ELISA that fucoidan is absorbed in the intestine only $0.6 \%$ [17].

Hydrogen, hydrophobic and sulphate bonds may be involved in maintaining the structure of the polysaccharide, which may change under the action of various compounds. Fucoidan degradation using hydrogen peroxide, ascorbic and hydrochloric acids [18] contributed to the formation of the 5th fraction: SC (coarse extract of fucoidan from S.Crassifolium), SCO (fraction obtained by processing with $\mathrm{H}_{2} \mathrm{O}_{2}$ ), SCA (fraction obtained by processing with ascorbic acid), SCOA (fraction obtained by processing of H2O2 and ascorbic acid) и SCH (fraction obtained by processing with hydrochloric acid). The decomposition of a large fucoidan molecule with such a way of processing led to an increase in the content of low molecular weight (HM) fractions of fucose monosaccharide. Fucoidan in in vitro studies on CaCo cells and in vivo on rat small intestine cells has a low absorption rate [19]. 
Methods for the purification of fucoidan affect both the structure and bioactivity of the obtained preparations. [20]. Thus, during the purification of the fucoidan preparation, there is an increase in fucose and sulphates and an increase in intestinal penetration into the blood. If the coarse fucoidan preparation advantagely was retained in the liver and spleen and did not penetrate the bloodstream poorly, the purified one is already detected in various organs and tissues; in particular in the kidneys and urine, i.e. incoming preparation is quite well excreted from the animal.

Currently sold fucoidan preparations are from various countries and companies derived from various types of algae, and in the annotation to the medicine there is often no indication of the type of algae, the percentage of fucoidan, its size (molecular weight), the content of fucose and sulphate groups. Therefore, when they are used, the expected health effects of the medicine are not always noted, which simply leads to discrediting of fucoidan.

Although fucoidan is a polysaccharide (food compound), it does not participate in the substrate provosion of metabolic processes (metabolism of proteins, fats, carbohydrates, vitamins and trace elements). Its bioactivity is due to the ability of the polysaccharide to influence the regulation of these processes through the effects on the nervous, hormonal, cytokine and signaling systems, i.e. Fucoidan exerts its bioactivity by acting on the body's regulatory systems.

During studying bioactivity, a unique side of fucoidan action was discovered - the ability to restore homeostasis (balance) of many processes in the body. Let's stop on some of them.

\section{Free radicals homeostasis}

Free radicals (FR) can be formed during exchange reactions and from the environment [21]. Accumulation of FR can lead to cell destruction, DNA, proteins and lipids damage. [22]. Antioxidants prevent such a negative effect of FR by reducing their level [23]. Therefore, a kind of myth that the use of antioxidants will preserve health and youth has arisen. However, the addition of synthetic antioxidants such as butylated hydroxytoluene and butylated hydroxyanisole in food contributed to the development of carcinogenesis and other negative manifestations [24-25].

Therefore, the question about finding natural products with antioxidant activity arose. In this regard, antioxidant vitamins A, E and C are widely used to "quench" FR, therefore herbal products with high antioxidant activity periodically appear on the market. But this is a half the truth, since free radicals are still necessary for some physiological processes, in particular for growth and adaptation, so the use of antioxidants in large quantities or very active can have a negative impact on these processes.

Fucoidan in this regard plays role not only as an antioxidant [26], but also as a pro-oxidant, i.e. if necessary, it will contribute to the formation of FR and improve the processes of growth and adaptation [27].

Thus, fucoidan contributes to maintaining the balance of the level of FR in the body under various physiological and pathological conditions due to antioxidant and prooxidant activities.

\section{Immune system homeostasis}

The next important balance that determines human health is the balance between the protective and attacking parts of the immune system. The protective element protects us from the influence of bacterial agents, viruses and cancer cells, while the attacking element or allergic element protects us against antigens.

Usually there is a decrease in the protective level of immunity, which leads to an increase in infectious diseases, viral infections and carcinogenesis. Therefore, the usual approach is the use of immunomodulators. This approach, on the one hand, can lead to the exhaustion of the protective element of the immune system, and, on the other hand, to the stimulation of the attacking element of the immune system. And, on the contrary, attempts to reduce the attacking level of immunity can lead to a deterioration of the defensive. That is why all medicine, restoring one link, can have a negative impact on the other, and we tell about immunomodulators negative manifestations.

Fucoidan helps the body fight infectious diseases through neutrophil activation [28]. It also has a stimulating effect on macrophages and dendritic cells. [29]. In addition, it is noted that fucoidan improves immunization in the elderly by increasing the level of antibodies [30].

Fucoidan has a clear anti-allergic effect. After subcutaneous administration of 2,4,6-trinitro chlorine benzol to mice an edema develops, then the value of it was decreased three times after receiving fucoidan at a dose of $200 \mu \mathrm{g}$ and six times 
at a dose of $400 \mu \mathrm{g}$ [31]. This effect of fucoidan is associated with the effect on galectin-9, since the administration of antibodies to galectin (or its inhibition) completely removed the effect of fucoidan. It is known that lactose (milk sugar) inhibits galactin; therefore, when it is administrated, suppression of the effect of fucoidan on the development of edema is also noted.

This must be taken into account when developing methods of preventing allergic reactions when using fucoidan. So the destruction of lactose by lactic acid bacteria is marked by an increase in the anti-allergic effect of fucoidan, which is observed when fucoidan is taken together with fermented milk products. [32].

\section{Homeostasis of the bone system}

Bones undergo constant renewal through synthesis and decay. With age, the amount of bone synthesis decreases and, on the contrary, their decay rate increases. This leads to a decrease in bone density (osteoporosis). Osteoporosis is a risk of fracture of the femoral neck (a common cause of death of older persons), physical instability, impaired quality of life, and requires the use of synthetic drugs, some of which have a negative effect. [33]. The process of bone formation is regulated by osteoblasts. Therefore, compounds that affect their function can help reduce osteoporosis.

Alkaline phosphatase (alkaline phosphatase) is an important component of bone formation, and its activation contributes to a local increase in phosphorus, protomer mineralization and a decrease in the concentration of extracellular pyrophosphate (mineralization inhibitor). Acceptance of fucoidan at a dose of $2 \mathrm{mg} / \mathrm{ml}$ helps to improve bone mineralization by activation alkaline phosphatase in 35\% [34].

Osteocalcin promotes bone formation, accelerating the growth of hydroxyapatite crystal [35]. Fucoidan increased the secretion of osteocalcin in 7F2 cells and the value of its mineralization in a dose-dependent manner.

In conclusion, it can be said that under the influence of fucoidan, there is a stimulation of osteogenic differentiation in vitro, and an increase in the anabolic effect on bone mineralization in vivo. This indicates that the use of fucoidan in the diet will prevent bone loss with age and increase its density.

\section{Homeostasis of the coagulation system}

Increased blood clotting is the risk of clot formation and vascular blockage, which in heart muscle leads to a heart attack and in brain tissue to the development of a stroke. There is a natural anticoagulant heparin in blood, which is also a sulfated polysaccharide, i.e. contains sulfate groupings.

Heparin is widely used in clinical practice, but its use can lead to complications in the form of hemorrhages. Fucoidan at a dose of $5 \mathrm{mg} / \mathrm{kg}$ increases the clotting time in mice [36]. The action of fucoidan is due to the activation of thromboplastin [37]. At the same time, fucoidan acts as an agonist for CLEC2 on platelets, causing them to aggregate [38].

It should be noted that fucoidan shows not only anticoagulant, but also procoagulant properties. [39]. Fucoidan in doses of 4-500 nM accelerate the clotting time in mice with hemophilia A or B [40].

Thrombography was used to evaluate the effect of fucoidan on clotting time. [41]. It is noted that NM fucoidan improved factor VIII homeostasis and reduced the manifestations of hemophilia.

Thus, fucoidan helps to maintain blood clotting homeostasis.

\section{Energy homeostasis}

The greatest attention in the literature is given to the ability of fucoidan to maintain homeostasis of carbohydrates and lipids, the violation of which is associated with the development of diabetes, obesity, and cardiovascular disorders.

The breadth of their spread should be attributed to epidemics of non-infectious origin (NCD). We have learned well how to deal with infectious diseases, which in the past were the main cause of low life expectancy. Now we have another problem - the lack of technology to struggle with NCD. 
The main disease of the violation of glucose homeostasis is diabetes mellitus (DM). There are lot of problems caused by diabetes, $80-95 \%$ of these cases refer to type 2 diabetes, in which the response of peripheral tissues to insulin is disturbed due to the development of insulin resistance (IR). The reason for the development of IR has not yet been established, which hinders the development of appropriate preventive and therapeutic measures.

According to the gloomy prognosis of diabetologists diabetes is not treated, and all that doctors can offer is a reduction of its negative manifestations. The basis of the pathological manifestations of diabetes is the development of hyperglycemia (an increase of sugar levels in blood above $6.5 \mathrm{mM}$ ), which leads to various functional disorders (glucotoxicity), therefore, all diabetologists' efforts are aimed at reducing the amount of carbohydrate intake from food and increasing the intensity of their absorption. Everything seemed to be simple - you just need to follow a diet (reduce the amount of carbohydrate intake) and carry out adequate physical activity (increase the speed of absorption), but, unfortunately, the number of people with diabetes is constantly increasing and you need to look for alternative ways to prevent and treat diabetes.

Fucoidan contributes to the reduction of the glycemic effect of starch-containing products by inhibiting amylase [42]. And at the same time, the $\mathrm{IC}_{\mathbf{5 0}}$ value (the concentration of the medicine that causes the enzyme inhibition by $50 \%$ ) was $103.83 \mu \mathrm{g}$ (microgram), which is much less than for acarbose (16 mg) - the pharmacological amylase inhibitor, so pharmacological effect of fucoidan is almost two times higher.

Inhibition of $\alpha$-glycosidase is an effective approach in the treatment of DM due to the reduction of glycemia. Fucoidan had an inhibitory effect on glycosidase (IC50 value was $67.9 \mu \mathrm{g} / \mathrm{ml}$ ) [43]. After fucoidan administration there was a decrease in glycemia and fasting glycated hemoglobin in $\mathrm{db} / \mathrm{db}$ mice. This study indicates the possibility of using fucoidan as a glycosidase inhibitor in DM.

Similar results were obtained [44] or fucoidan from F.vesiculosus, when the IC50 value was $49 \mu \mathrm{g} / \mathrm{ml}$.

DM is a multisystem disease characterized by impaired activity of organs and systems. Control of postprandial (after a meal) glycemia is an important factor in the prevention and treatment of DM. The administration of fucoidan at a dose of $200 \mathrm{mg} / \mathrm{kg}$ to a $\mathrm{db} / \mathrm{db}$ mice with diabetes showed a positive effect in terms of the glucose-tolerant test, the amount of food intake, the change in body weight, the concentration of fasting glucose and biochemical indicators [45].

A randomized study with double placebo control of the effect of $0.5 \mathrm{~g}$ fucoidan administration was performed on 25 obese volunteers [46]. Thirteen individuals received fucoidan daily before breakfast, and 12 received a placebo. Then all were evaluated for glucose, cholesterol (cholesterol), HDL cholesterol, LDL cholesterol (LDL-C), triglycerides, and insulin, and glucose homeostasis was assessed by the HOMA test (glucose tolerance test). Fucoidan administration contributed to a decrease in diastolic pressure $(71.7 \pm 12.2$ vs. $67.8 \pm 13.8 \mathrm{mmHg} ; \mathrm{P}<0.05)$ and LDL-C $(3.1 \pm 0.5$ vs. $2.7 \pm 0.6$ $\mathrm{mmol} / \mathrm{l} ; \mathrm{P}<0.01)$, an increase in insulin level $(60.6 \pm 24.0$ vs. $78.6 \pm 32.4 \mathrm{pmol} / \mathrm{l} ; \mathrm{P}<0.05)$, HOMA $\beta$-cell $(35.0 \pm 20.8$ vs. $50.6 \pm 18.7 ; \mathrm{P}<0.05)$. It has been concluded that fucoidan administration for obese individuals for 3 months reduces pressure and LDL-C levels, but increasing insulin secretion. Although the authors write that they monitored obese individuals, obesity usually shows signs of type 2 diabetes.

A decrease of glucose level in blood of laboratory animals was also noted in type 1 diabetes. The mechanism of such influence is explained by the authors [47] due to the blocking of negative manifestations of oxidative stress by fucoidan as one of the causes of glucotoxicity.

Fucoidan due to its antioxidant properties, prevents the development of hypertension (aortic tone increase) and hyperlipidemia. Indeed, diabetics have a high risk of endothelial dysfunction and vascular damage due to hyperglycemia, lipidemia, and oxidative stress. Atherosclerosis, hypertension and peripheral vascular disorders are characteristic of these patients. Endothelial nitrite oxidase (NO) has been found to regulate vascular homeostasis through vasadilation and the protection of the effects of damaging agents. A decrease in NO leads to an increase in proinflammatory factors, such as nuclear factor kappa B, and further increases in endothelial inflammation and atherosclerosis. Therefore, the improvement of NO-functioning is a target (by the mechanism of action) for endothelium and reduction of vascular disorders in diabetic rats.

In recent years there is a great importance of lipid metabolism in the mechanism of diabetes, when lipids act as a competitor for the process of energy formation and thereby inhibit glucose uptake (Randle cycle). Therefore, it was very interesting to investigate the effect of fucoidan on lipid metabolism in diabetes. AMP activated protein kinase (AMPK) play a main role in the regulation of glucose uptake by the muscles, and is activated under stress and is inhibited by insulin. 
In this regard, the in vitro study in microtubules (L6 myotubes) and in vivo study in mice with DM are of big interest [48]. Studies of the fucoidan effect conducted in comparison with the first-line medicine - metmorphine. In this regard, this work also reveals the mechanism of the positive effect of metmorphine in diabetes.

\section{The effect of fucoidan on regulatory systems}

Hormones are key regulators of the rate of metabolic processes. Usually, all hormones work in pairs, i.e. some accelerate certain reactions, while others, on the contrary, reduce. In women of fertile age, there is an increase in the ratio between the levels of estrogen and progesterone, which is the cause of the development of endometriosis, breast and ovarian cancer, and infertility. Pilot study on women with impaired menstrual cycle and endometriosis [49] showed that fucoidan administration for four months at a dose of 0.7 and $1.4 \mathrm{~g} /$ day contributed to the normalization of the level and ratio between estradiol and progesterone. At the same time, a recovery to the normal duration of the menstrual cycle and days of menstruation was noted. The effect of fucoidan was manifested in a dose-dependent manner.

But there is a greatest interest of the positive effect of fucoidan on the nervous system. Fucoidan prevents the death of dopamine receptors [50]. Parkinson's disease was modeled by 1-methyl-4-phenylpyridinium (MPTP) administration on C57/BL mice моделировали болезнь Паркинсона введением 1-methyl-4-phenylpyridinium (MPTP) and it was showed that fucoidan reduced behavioral disorders, increased the level of dopamine in the striatal and its metabolic level, and reduced cell death. A clear increase in tyrosine hydroxylase expression was also noted. In addition, fucoidan suppressed MPTP caused lipid peroxidation, antioxidant enzymes activity decrease and the death of MN9D cells.

The deposition of amyloid protein (A beta), a peptide of 39-43 AK, in the brain and the loss of cholinergic neurons lie in the pathogenesis of Alzheimer's disease. Fucoidan in a dose-dependent manner $(0.1-1.0 \mu \mathrm{M})$ reduced cell death by $16 \%$ and the death of cholinergic neurons by 40-60\% [51].

Fucoidan prevents the negative effects of $A \beta$ amyloid on rat learning and behavior. In addition, fucoidan reduced the activity of choline acetyl transferase, superoxide dismutase, glutathione peroxidase, reduced acetylcholine amount and increased the activity of cholinesterase in the hippocampus [52]. The results show that fucoidan improved learning and memory in treated with $A \beta$ rats by regulating the cholinergic system, reducing oxidative stress and inhibiting cell apoptosis.

The amount of amyloid elimination from the brain decreases dramatically after insulin administration, and at a dose of $100 \mu \mathrm{g} / \mathrm{ml}$, the elimination is halved, so such hormone administration to diabetics can contribute to the development of dimentia [53]. Therefore, for patients with diabetes, fucoidan is an important component of functional nutrition.

Environmental pollution contributes to an increase in toxic compounds in the brain and dopamine neurons damage [54]. The smaller the contaminating particle in size, the easier it can penetrate the brain and cause toxic manifestations, as shown by the level of peroxides in the brain. Stay at an environmentally unfriendly environment leads to an increase in the formation of FR (free radicals) in the neuroglia and further development of manifestations of oxidative stress. Therefore, the use of antioxidants is an important aspect of prevention against the development of CNS disorders. Associated with this there is the positive effect of fucoidan in preventing the development of Parkinson's disease by reducing the manifestations of oxidative stress [55]. The fucoidan administration reduced cell apoptosis by increasing the level/activity of GSH, GSH-PX, MDA and CAT in MPTP mice.

Prolonged use of fucoidan prevents the development of neuronal disorders caused by the administration of 6hydroxydopamine (6-OHDA) n rats when modeling Parkinson's disease [56], which is associated with a decrease in the loss of dopamine neurons and dopamine fibers in the striatum.

Activation of microglia and damage to dopamine neurons is observed with the lipopolysaccharide administration [57]. Fucoidan improved cognitive functions and prevented the loss of dopamine neurons, as well as the activation of microglia in nigra substance in rats, and there was a decrease in the production of TNF- $\alpha$ and FR in microglia. Lipopolysaccharides transform the cell shape, and fucoidan prevents this change at a dose of $62.5 \mu \mathrm{g} / \mathrm{ml}$. However, at a dose of $125 \mu \mathrm{g} / \mathrm{ml}$, fucoidan inhibits the production of NO by microglia by $75 \%$ and reduces the expression of iNOS mRNA and protein by $50 \%$. Fucoidan inhibits p38 and ERK kinase by $50 \%$.

Neutrophils infiltration and the development of neurological disorders observed with the antibiotics administration with bacterial meningitis. The fucoidan administration prevented the negative effect of antibiotics due to leukocytes migration into the cerebrospinal fluid (CSF) and into the brain. Fucoidan administration at a dose of $10 \mathrm{mg} / \mathrm{kg}$ every 
two hours showed a decrease in leukocyte level [58]. This aspect must be considered during antibiotic therapy for meningitis to reduce mortality in pneumococcal meningitis.

It is well known that glial cells respond to inflammatory processes with the production of various bioactive molecules. One of these molecules is nitrogen nitrite (NO). Although physiologically, NO acts as a neuromodulator and neurotransmitter in the brain, but when it is abundant, the normal function of glial cells is disrupted, leading to damage and death. NO production is caused by overexpression of inducible isoforms of the NO synthase enzyme (iNOS) in glial cells [59].

Repeated restraint stress in rodents increases depression-like behavior and increases corticotropin-releasing factor expression in the hypothalamus. Male rats were received 10,20 , or $50 \mathrm{mg} / \mathrm{kg}$ of fucoidan peritoneally 30 minutes before stress for 14 days [60]. There was a decrease in stress-induced behavior. Therefore, fucoidan may be a useful agent for reducing the complex of symptoms of depression, which may help reduce the consumption of antidepressants.

Ischemic stroke is one of the frequent causes of death, so the development of preventive measures is very important. To study it, the most frequently used model is a clamping and subsequent restoration (reperfusion) of vessel circulation. There is a development of inflammation in the affected area [61], FR increase [62] and apoptosis [63]. Fucoidan reduces all these negative manifestations, so its administration led to a reduction in the negative impact of ischemia [64].

Leukocyte infiltration into the ischemic zone is the most important cause of organ damage. Fucoidan in a dosedependent manner reduced neurological manifestations by reducing the leukocytes adhesion and can be used to treat ischemic encephalopathy [65].

Hemorrhagic stroke is one of the fatal diseases due to treatment failure. Hematoma is the main pathogenetic cause of brain damage. In CD-1 male mice, a hemorrhagic stroke was induced by the introduction of bacterial collagenase [66]. Fucoidan administration at a dose of 25 and $100 \mathrm{mg} / \mathrm{kg}$ contributed to the reduction of neurological manifestations. Such results were obtained by other authors [67]. In rats treated with fucoidan, coagulation and hemodeliuation were impaired, that increased the hematoma, but inflammation around the hematoma decreased after three days. There is was a rapid improvement in motor function in the first week after hemorrhage and a rapid recovery of the passive motor test.

Traumatic brain injury (TBI) is often incompatible with life. 16-18 months aged mice were given fucoidan at a 10-50 $\mathrm{mg} / \mathrm{kg}$, that reduced the amount of brain cells breakdown and decreased neurological manifestations [68]. Fucoidan reduced protein carbonylation, lipid peroxidation and FR generation, as well as mitochondrial dysfunction. The obtained data indicate the neuroprotective properties of fucoidan.

\section{Conclusion}

Fucoidan is a wide range of nutraceuticals, which helps maintain homeostasis of the antioxidant, anticoagulant, bone and immune systems. It maintains the homeostasis of energy sources (glucose and fatty acids). Its influence is largely due to the ability of the polysaccharide to improve the regulatory properties of the neuro-hormonal level of regulation that promote health, improve quality and longevity of a person.

\section{Compliance with ethical standards}

\section{Disclosure of conflict of interest}

Authors declare that the research was conducted in the absence of any commercial or financial relationships that could be construed as a potential conflict of interest.

\section{References}

[1] Kadam SU and Prabhasankar P. (2010).Marine foods as functional ingredients in bakery and pasta products. Food Res. Int, 43, 1975-1980.

[2] Iso H and Kubota Y. (2007). Nutrition and disease in the Japan Collaborative Cohort Study for Evaluation of Cancer (JACC). Asian Pac. J. Cancer Prev, 8, 35-80.

[3] Kylin H. (1913). Biochemistry of sea algae. H. Z.Physiol.Chem, 83, 171-197. 
[4] Kylin H. (1915). Analysis of the biochemistry of the seaweed. H. Z. Physiol. Chem, 94, 337-425.

[5] Hoagland DR and Lieb LL. (1915). The complex carbohydrates and forms of sulphur in marine algae of the Pacific coast. J. Biol. Chem, 23, 287-297.

[6] Li B, Lu F, Wei X and Zhao R. (2008).Fucoidan: Structure and bioactivity. Molecules, 13, 1671-1695.

[7] Morya VK, Kim J and Kim EK. (2012). Algal fucoidan: Structural and size-dependent bioactivities and their perspectives. Appl. Microbiol. Biotechnol, 93, 71-82.

[8] Fitton JH, Stringer DN and Karpiniec SS. (2015). Therapies from Fucoidan: An Update. Mar. Drugs, 13, 5920-5946.

[9] Ale MT., Maruyama H, Tamauchi H, Mikkelsen JD and Meyer AS. (2011). Fucose-Containing Sulfated Polysaccharides from Brown Seaweeds Inhibit Proliferation of Melanoma Cells and Induce Apoptosis by Activation of Caspase-3 in Vitro Mar Drugs, 9, 2605-2621.

[10] Marudhupandi T, et al. (2014). In vitro antioxidant properties of Fucoidan fractions from Sargassum tenerrimum. Pak J Biol Sci., 17, 402-407.

[11] Li B, Lu F, Wei X and Zhao R. (2008). Fucoidan: Structure and bioactivity. Molecules, 13, 1671-1695.

[12] CY Huang, et al. (2018). Antibacterial and Antioxidant Capacities and Attenuation of Lipid Accumulation in 3T3L1 Adipocytes by Low-Molecular-Weight Fucoidans Prepared from Compressional-PuffingPretreated Sargassum Crassifolium Mar Drugs, 16-24.

[13] Koyanagi S, Tanigawa N, Nakagawa H, Soeda S and Shimeno H. (2003). Oversulfation of fucoidan enhances its anti-angiogenic and antitumor activities. Biochem Pharmacol., 65, 173-179.

[14] Anno K. (1970). Isolation of L-fucose 4-sulfate from fucoidan. Carbohydr. Res, 13, 167-169.

[15] Patankar MS, Oehninger S, Barnett T, Williams RL and Clark GF. (1993).A revised structure for fucoidan may explain some of its biological activities. J.Biol Chem, 268, 21770-21776.

[16] Tokita Y. (2010). Development of a fucoidan-specific antibody and measurement of fucoidan in serum and urine by sandwich ELISA. Biosci. Biotechnol. Biochem, 74, 350-357.

[17] CY Huang. (2018). Antibacterial and Antioxidant Capacities and Attenuation of Lipid Accumulation in 3T3-L1 Adipocytes by Low-Molecular-Weight Fucoidans Prepared from Compressional-Puffing-Pretreated Sargassum Crassifolium Mar Drugs, 16-24.

[18] T Nagamine. (2015).Intestinal Absorption of Fucoidan Extracted from the Brown Seaweed, Cladosiphon okamuranus Mar Drugs, 13, 48-64.

[19] P Saboural, F Chaubet, F Rouzet et al. (2014). Purification of a Low Molecular Weight Fucoidan for SPECT Molecular Imaging of Myocardial Infarction Mar Drugs, 12, 4851-4867.

[20] Wang J, Zhang Q, Song H and Li P. (2010).Potential antioxidant and anticoagulant capacity of low molecular weight fucoidan fractions extracted from Laminaria japonica. Int. J. Biol. Macromol, 46, 6-12.

[21] Sallmyr A, Fan J and Rassool FV. (2008).Genomic instability in myeloid malignancies: Increased reactive oxygen species (ROS), DNA double strand breaks (DSBs) and error-prone repair. Cancer Lett, 270, 1-9.

[22] Halliwell B. (1996). Antioxidants in human health and disease. Annu. Rev. Nutr, 16, 33-50.

[23] Branen AL. (1975). Toxicology and biochemistry of butylated hydroxyanisole and butylated hydroxytoluene. J. Am. Oil Chem. Soc, 52, 59-63.

[24] Ito N, et al. (1983). Carcinogenicity of butylated hydroxyanisole in F344 rats. J. Natl. Cancer Inst, 70, 343-352.

[25] Marudhupandi T. (2014). In vitro antioxidant properties of Fucoidan fractions from Sargassum tenerrimum. Pak.J.Biol.Sci., 17, 402-407.

[26] C Wei. (2015). Fucoidan inhibits proliferation of the SKM-1 acute myeloid leukaemia cell line via the activation of apoptotic pathways and production of reactive oxygen species Mol Med Rep, 12, 6649-6655.

[27] Jin JO and Yu Q. (2015). Fucoidan delays apoptosis and induces pro-inflammatory cytokine production in human neutrophils. Int J Biol Macromol., 73, 65-71.

[28] SY Kim and H-G Joo. (2015). Evaluation of adjuvant effects of fucoidan for improving vaccine efficacy J Vet Sci, 16, 145-150. 
[29] H Negishi, Mori M, Mori H and Yamori Y. (2013). Supplementation of Elderly Japanese Men and Women with Fucoidan from Seaweed Increases Immune Responses to Seasonal Influenza Vaccination J Nutr, 143, $1794-1798$.

[30] Tanino Y, Hashimoto T, Ojima T and Mizuno M. (2016). F-fucoidan from Saccharina japonica is a novel inducer of galectin-9 and exhibits anti-allergic activity J Clin Biochem Nutr, 59, 25-30.

[31] Kawashima T, Murakami K, Nishimura I, Nakano T and Obata A. (2012). A sulfated polysaccharide, fucoidan, enhances the immunomodulatory effects of lactic acid bacteria. Int J Mol Med., 29, 447-53.

[32] Genant HK, Baylink DJ and Gallagher JC. (1989). Estrogens in the prevention of osteoporosis in postmenopausal women. Am J Obstet Gynecol, 161, 1842-1846.

[33] Hwang PA. (2015). The in vitro and in vivo effects of the low molecular weight Fucoidan on the bone osteogenic differentiation properties. Cytotechnjlogy, 14.

[34] Boskey AL. (1998). Fourier transform infrared microspectroscopic analysis of bones of osteocalcin-deficient mice provides insight into the function of osteocalcin. Bone, 23, 187-196.

[35] Min SK, Kwon OC, Lee S, Park KH and Kim JK. (2011). An antithrombotic fucoidan, unlike heparin, does not prolong bleeding time in a murine arterial thrombosis model: A comparative study of undaria pinnatifida sporophylls and fucus vesiculosus. Phytother. Res, 26, 752-757.

[36] Jin WA. (2013). comparative study of the anticoagulant activities of eleven fucoidans. Carbohydr. Polym, 91, 1-6.

[37] Manne BK, Getz TM, Hughes CE, Alshehri O, Dangelmaier C, Naik UP, Watson SP and Kunapuli SP. (2013). Fucoidan is a novel platelet agonist for the c-type lectin-like receptor 2 (clec-2) J. Biol. Chem, 288, 7717-7726.

[38] Zhang Z, Till S, Jiang C, Knappe S, Reutterer S, Scheiflinger F, Szabo CM and Dockal M. (2014). Structure-activity relationship of the pro- and anticoagulant effects of fucus vesiculosus fucoidan. Thromb. Haemost, 111, 429-437.

[39] Liu T, Scallan CD, Broze GJ. Patarroyo-White S, Pierce GF, Johnson KW. (2006). Improved coagulation in bleeding disorders by Non-Anticoagulant Sulfated Polysaccharides (NASP). Thromb Haemost., 95, 68-76.

[40] Zhang Z, Till S, Knappe S, Quinn C, Catarello J, Ray GJ, Scheiflinger F, Szabo CM and Dockal M. (2015). Screening of complex fucoidans from four brown algae species as procoagulant agents. Carbohydr Polym., 115, 677-85.

[41] Lakshmana SS, Vinoth KT, Geetharamani D, Suja G, Yesudas R and Chacko A. (2015). Fucoidan - An $\alpha$-amylase inhibitor from Sargassum wightii with relevance to NIDDM. Int.J.Biol.Macomol, 81, 644-7.

[42] Shan X, Liu X, Hao J, Cai C, Fan F, Dun Y, Zhao X, Liu X, Li C and Yu G. (2016). In vitro and in vivo hypoglycemic effects of brown algal fucoidans. Int.J.Biol.Macomol, 82, 249-55.

[43] Kim KT, Rioux LE and Turgeon SL. (2014).Alpha-amylase and alpha-glucosidase inhibition is differentially modulated by Fucoidan obtained from Fucus vesiculosus and Ascophyllum nodosum. Phytochemistry., 98, 2733.

[44] Kim KJ, Yoon KY and Lee BY. (2012). Fucoidan regulate blood glucose homeostasis in C57BL/KSJ m+/+db and C57BL/KSJ db/db mice. Fitoterapia, 83, 1105-1109.

[45] Hernández-Corona DM, Martínez-Abundis E and González-Ortiz M. (2014). Effect of Fucoidan administration on insulin secretion and insulin resistance in overweight or obese adults. J Med Food, 17, 830-842.

[46] Liang Z, Zheng Y, Wang J, Zhang Q, Ren S, Liu T, Wang Z and Luo D. (2016).Low molecular weight Fucoidan ameliorates streptozotocin-induced hyper-responsiveness of aortic smooth muscles in type 1 diabetes rats. J.Ethnopharmacol, 15, 191, 341-349.

[47] Jeong YT, Kim YD, Jung YM, Park DC, Lee DS, Ku SK, Li X, Lu Y, Chao GH, Kim KJ, Lee JY, Baek MC, Kang W, Hwang SL and Chang HW. (2013). Low Molecular Weight Fucoidan Improves Endoplasmic Reticulum Stress-Reduced Insulin Sensitivity through AMP-Activated Protein Kinase Activation in L6 Myotubes and Restores Lipid Homeostasis in a Mouse Model of Type 2 Diabetes Molecular Pharmacology, 84, 147-157.

[48] CF Skibola. (2004). The effect of Fucus vesiculosus, an edible brown seaweed, upon menstrual cycle length and hormonal status in three pre-menopausal women: a case report BMC Complement Altern Med, 4, 10.

[49] Luo D, et al. (2009). Fucoidan protects against dopaminergic neuron death in vivo and in vitro. Eur.J. Pharmacol, $617,33-40$.

[50] Jhamandas JH, Wie MB, Harris K, MacTavish D and Kar S. (2005). Fucoidan inhibits cellular and neurotoxic effects of beta-amyloid (A beta) in rat cholinergic basal forebrain neurons. Eur.J.Neurosci, 21, 2649-2659. 
[51] Gao Y. (2012). Fucoidan, a sulfated polysaccharide from brown algae, improves cognitive impairment induced by infusion of $A \beta$ peptide in rats. Environ Toxicol Pharmacol., 33, 304-11.

[52] T Shiiki, S Ohtsuki, A Kurihar, H Naganuma, K Nishimura, M Tachikawa, K Hosoya and T Terasaki. (2004). Brain Insulin Impairs Amyloid- $\beta(1-40)$ Clearance from the Brain The Journal of Neuroscience, 24, 9632-9637.

[53] S Levesque et al. (2013). The Role of MAC1 in Diesel Exhaust Particle-induced Microglial Activation and Loss of Dopaminergic Neuron Function J Neurochem, 125, 756-765.

[54] Wang J, Wang X., Yi K. and Zhao Y. (2016). Structure-activity relationship of sulfated hetero/galactofucan polysaccharides on dopaminergic neuron. Int.J.Biol.Macromol., 82, 878-883.

[55] Zhang FL, et al. (2014). Therapeutic effects of fucoidan in 6-hydroxydopamine-lesioned rat model of Parkinson's disease: Role of NADPH oxidase-1. CNS Neurosci Ther., 20, 1036-1044.

[56] Cui YQ, Jia YJ, Zhang T, Zhang QB and Wang XM. (2012). Fucoidan protects against lipopolysaccharide-induced rat neuronal damage and inhibits the production of proinflammatory mediators in primary microglia. CNS Neurosci Ther., 18, 827-833.

[57] C Granert, J Raud and L Lindquist. (1998). The Polysaccharide Fucoidin Inhibits the Antibiotic-Induced Inflammatory Cascade in Experimental Pneumococcal Meningitis Clin Diagn Lab Immunol, 5, 322-324.

[58] Ghasemi M and Fatemi A. (2014).Pathologic role of glial nitric oxide in adult and pediatric neuroinflammatory diseases. Neurosci Biobehav Rev., 45, 168-82.

[59] Lee B, Shim I, Lee H and Hahm DH. (2013). Fucoidan prevents depression-like behavior in rats exposed to repeated restraint stress. J Nat Med., 67, 534-544.

[60] Jean WC, et al. (1998). Reperfusion injury after focal cerebral ischemia: the role of inflammation and the therapeutic horizon. Neurosurgery, 43, 1382-1396.

[61] Sugawara T and Chan PH. (2003). Reactive oxygen radicals and pathogenesis of neuronal death after cerebral ischemia. Antioxid Redox Signal, 5, 597-607.

[62] Duan Q, et al. (2004). Role of mitochondria in neuron apoptosis during ischemia-reperfusion injury. J Huazhong Univ Sci Technol Med Sci, 24, 441-444.

[63] N Che, Y Ma and Y Xin. (2017). Protective Role of Fucoidan in Cerebral Ischemia-Reperfusion Injury through Inhibition of MAPK Signaling Pathway Biomol Ther (Seoul), 25, 272-278.

[64] Uhm CS, et al. (2003). Effective treatment with fucoidin for perinatal hypoxic-ischemic encephalopathy in rats. Neurosci Lett., 353, 21-24.

[65] Burchell SR et al. (2016). Fucoidan from Fucus vesiculosus Fails to Improve Outcomes Following Intracerebral Hemorrhage in Mice. Acta Neurochir Suppl., 121, 191-198.

[66] Del Bigio MR, et al. (1999). Effect of fucoidan treatment on collagenase-induced intracerebral hemorrhage in rats. Neurol Res., 21, 415-419.

[67] Wang T, et al. (2016). Low-Molecular-Weight Fucoidan Attenuates Mitochondrial Dysfunction and Improves Neurological Outcome After Traumatic Brain Injury in Aged Mice: Involvement of Sirt3. Cell Mol Neurobiol., 36, 1257-1268.

\section{How to cite this article}

Emil M and Victor K. (2020). Fucoidan: A nutraceutical for metabolic and regulatory systems homeostasis maintenance. World Journal of Advanced Research and Reviews, 6(1), 255-264. 